\title{
Efficient structural sandwich wall panels devoid of thermal bridges
}

\begin{abstract}
Reinforced concrete sandwich wall panels are developed to reduce the effect of thermal transmission across the wall systems. The reduction of the thermal transmission is achieved through incorporation of an insulating layer. However, this insulating layer led to a reduction of structural performance. The provision of shear connection in the sandwich system improved its structural integrity and increased with increase in a number of shear connectors. However, if the shear connectors are placed directly across the layers of the concrete wythes, it will decrease its thermal efficiency. The thermal and structural performance works in contrary effect to an increasing number of shear connectors. Hence, optimizing both structural and thermal efficiencies simultaneously in reinforced concrete sandwich system has been a challenge for a very long time. Therefore, this paper presents an alternative approach focusing on the thermal path method to produce an optimum shear connector used. This approach eliminates the direct transmission path between the two wythes and, at the same time, avoids the use of alternative materials such as fibre-reinforced polymers which could be uneconomical. With this method, both thermal and structural efficiencies are optimized using only conventional concrete and steel materials.
\end{abstract}

Keyword: Precast concrete sandwich panel; Shear connection; Thermal performance 